\title{
Higher-order topological superconductivity of spin-polarized fermions
}

\author{
Junyeong Ahn (i) and Bohm-Jung Yang* \\ Center for Correlated Electron Systems, Institute for Basic Science (IBS), Seoul 08826, Korea; \\ Department of Physics and Astronomy, Seoul National University, Seoul 08826, Korea; \\ and Center for Theoretical Physics (CTP), Seoul National University, Seoul 08826, Korea
}

(Received 2 July 2019; revised manuscript received 4 December 2019; accepted 20 February 2020; published 12 March 2020)

\begin{abstract}
We study the superconductivity of spin-polarized electrons in centrosymmetric ferromagnetic metals. Due to the spin polarization and the Fermi statistics of electrons, the superconducting pairing function naturally has odd parity. According to the parity formula proposed by Fu, Berg, and Sato, odd-parity pairing leads to conventional first-order topological superconductivity when a normal metal has an odd number of Fermi surfaces. Here, we derive generalized parity formulas for the topological invariants characterizing the higher-order topology of centrosymmetric superconductors. Based on the formulas, we systematically classify all possible band structures of ferromagnetic metals that can induce inversion-protected higher-order topological superconductivity. Among them, doped ferromagnetic nodal semimetals are identified as the most promising normal-state platform for higher-order topological superconductivity. In two dimensions, we show that odd-parity pairing of doped Dirac semimetals induces a second-order topological superconductor. In three dimensions, odd-parity pairing of doped nodal-line semimetals generates a nodal-line topological superconductor with monopole charges. On the other hand, odd-parity pairing of doped monopole nodal-line semimetals induces a three-dimensional third-order topological superconductor. Our theory shows that the combination of superconductivity and ferromagnetic nodal semimetals opens up another avenue for future topological quantum computations using Majorana zero modes.
\end{abstract}

DOI: 10.1103/PhysRevResearch.2.012060

Introduction. Recently, odd-parity superconductivity has received great attention due to its potential to realize topological superconductors (TSCs) [1-5]. Fu and Berg [6] and also Sato $[7,8]$ proposed a simple but powerful parity formula relating the parity configuration in the normal state and the topological property of the odd-parity superconducting state. The simplicity of the formula allows a fast diagnosis of the topological nature of a superconducting state by just counting the number of Fermi surfaces, which greatly facilitates the search for TSCs in centrosymmetric materials.

One limitation of the Fu-Berg-Sato formula is that it can be applied only to conventional first-order TSCs in which $d$ dimensional bulk topology supports gapless Majorana states on $(d-1)$-dimensional boundaries. However, recent studies on topological crystalline phases have uncovered higher-order TSCs whose $d$-dimensional bulk topology protects gapless Majorana fermions on the boundaries with dimensions lower than $(d-1)$ [9-14]. In general, $k$ th-order TSCs in $d$ dimensions host $(d-k)$-dimensional boundary Majorana states. In the case of $d$ th-order TSCs in $d$ dimensions, Majorana zero

\footnotetext{
*bjyang@snu.ac.kr

Published by the American Physical Society under the terms of the Creative Commons Attribution 4.0 International license. Further distribution of this work must maintain attribution to the author(s) and the published article's title, journal citation, and DOI.
}

modes (MZMs) exist at the corners, which can be potentially useful for topological quantum computations [2-5].

Up to now, several interesting ideas have been proposed to realize two-dimensional (2D) second-order TSCs in various different settings, such as using the superconducting proximity effect on quantum Hall insulators [15], quantum spin Hall insulators [13,16,17], second-order topological insulators [18], Rashba semiconductors [19], and nanowires [20], breaking time-reversal symmetry of TSCs with helical Majorana edge states by applying an external magnetic field [9,2124] or attaching antiferromagnets [25], and some other ideas $[13,23,26-28]$. In 3D, on the other hand, there are only a few mechanisms proposed for realizing a third-order TSC such as applying a magnetic field to a 3D second-order TSC with helical hinge modes [9]. For more systematic investigations of higher-order TSCs, a simple criterion for diagnosing higher-order band topology, similar to the Fu-Berg-Sato parity formula for first-order TSCs, is highly desired. Although some formulas for higher-order TSCs having gapless boundary states were proposed recently [29], the parity formula for $d$ th-order TSCs hosting MZMs is still lacking.

In this Rapid Communication, we establish generalized parity formulas for higher-order TSCs and apply them to ferromagnetic metals where odd-parity superconductivity naturally arises. Using the generalized parity formulas, we classify all possible spin-polarized band structures of centrosymmetric ferromagnetic metals that can realize inversion-protected higher-order TSC. From this analysis, we find doped ferromagnetic nodal semimetals as an ideal normal state that 
realizes higher-order TSCs. Explicitly, in 2D, odd-parity pairing of a doped Dirac semimetal (DSM) induces a 2D secondorder TSC. In 3D, odd-parity pairing of a doped nodal-line semimetal (NLSM) generates a nodal-line superconductor with monopole charges. Furthermore, in the case of a doped monopole NLSM [30,31], odd-parity pairing induces a 3D third-order TSC. These findings show that the combination of superconductivity and spin-polarized 2D and 3D nodal semimetals can be promising platforms for topological quantum computations using MZMs.

Symmetry and nodal structures. Let us first clarify the symmetry of the normal and superconducting states of ferromagnetic metals with inversion symmetry $P_{0}$ and classify the relevant nodal structures. We assume that an electron's spin is polarized along the $z$ direction. Also, we neglect spin-orbit coupling, but its influence is discussed later. In this setting, although time-reversal symmetry $\mathcal{T}=i \sigma_{y} K$ is broken, the ferromagnetic metallic state is symmetric under the effective time reversal $T \equiv e^{i \pi \sigma_{y} / 2} \mathcal{T}=K$ defined as the product of $\mathcal{T}$ and a $180^{\circ}$ spin rotation around the $y$ axis, $e^{i \pi \sigma_{y} / 2}$. Here, $\sigma_{y}$ is a Pauli matrix for spin degrees of freedom, and $K$ denotes the complex conjugation operator. Also, $P_{0}=P_{0}^{*}$ because $\left[P_{0}, T\right]=0$. Then, the system is invariant, locally at each momentum $\mathbf{k}$, under $P_{0} T$ symmetry satisfying $\left(P_{0} T\right)^{2}=1$. Such a $P_{0} T$-symmetric system belongs to the $\mathbf{k}$-local symmetry class $\mathrm{AI}+\mathcal{I}$ proposed by Bzdusek and Sigrist [32], where the $1 \mathrm{D}$ and $2 \mathrm{D}$ topological phases are classified by $Z_{2}$ invariants $[30,32]$. Here, the $1 \mathrm{D} Z_{2}$ invariant is the quantized Berry phase, which is the topological charge of $2 \mathrm{D}$ Dirac points and also of $3 \mathrm{D}$ nodal lines. The $2 \mathrm{D} Z_{2}$ invariant is the monopole charge of 3D nodal lines.

To describe the superconducting state, we introduce a $2 \mathrm{~N}$ component Nambu spinor $\hat{\Psi}(\mathbf{k})=\left[\hat{c}_{\uparrow \alpha}(\mathbf{k}), \hat{c}_{\uparrow \beta}^{\dagger}(\mathbf{k})\right]^{T}$, where $\hat{c}_{\uparrow \alpha}(\mathbf{k})\left[\hat{c}_{\uparrow \alpha}^{\dagger}(\mathbf{k})\right]$ is an electron creation (annihilation) operator with spin up and the orbital indx $\alpha=1, \ldots, N$. The corresponding Bogoliubov-de Gennes (BdG) Hamiltonian can be written as $\hat{H}=\hat{\Psi}^{\dagger} H_{\mathrm{BdG}} \hat{\Psi}$, where

$$
H_{\mathrm{BdG}}=\left(\begin{array}{cc}
h(\mathbf{k}) & \Delta(\mathbf{k}) \\
\Delta^{\dagger}(\mathbf{k}) & -h^{T}(-\mathbf{k})
\end{array}\right) .
$$

Here, $h(\mathbf{k})$ indicates the Hamiltonian for the normal state, and the pairing function $\Delta_{\alpha \beta}(\mathbf{k})$ with orbital indices $\alpha, \beta$ satisfies $\Delta_{\alpha \beta}(\mathbf{k})=-\Delta_{\beta \alpha}(-\mathbf{k})$ because of the Fermi statistics of electrons. Since the pairing function forms an irreducible representation of the symmetry group, it can have either odd parity $P_{0} \Delta(\mathbf{k}) P_{0}^{-1}=-\Delta(-\mathbf{k})$ or even parity $P_{0} \Delta(\mathbf{k}) P_{0}^{-1}=$ $+\Delta(-\mathbf{k})$.

In the weak-pairing limit, we can focus on the pairing at the Fermi energy $E_{F}$ and define the corresponding pairing function as $\Delta_{E_{F}}(\mathbf{k})$. Then, $P_{0} \Delta_{E_{F}}(\mathbf{k}) P_{0}^{-1}=\Delta_{E_{F}}(\mathbf{k})$ because $\Delta_{E_{F}}$ is a $1 \times 1$ matrix. The Fermi statistics $\Delta_{E_{F}}(\mathbf{k})=-\Delta_{E_{F}}(-\mathbf{k})$ naturally shows that the pairing function satisfies the oddparity condition

$$
P_{0} \Delta_{E_{F}}(\mathbf{k}) P_{0}^{-1}=-\Delta_{E_{F}}(-\mathbf{k}) .
$$

Therefore, in Eq. (1), we consider only odd-parity pairing functions that satisfy $P_{0} \Delta(\mathbf{k}) P_{0}^{-1}=-\Delta(-\mathbf{k})$ (see also the Supplemental Material [33]). The corresponding odd-parity BdG Hamiltonian is symmetric under inversion $P=\tau_{z} P_{0}$ which anticommutes with the particle-hole symmetry $C=$ $\tau_{x} K$, where $\tau_{x, y, z}$ are Pauli matrices for the Nambu space. $P T$ and $C P$ symmetries satisfy $(P T)^{2}=1$ and $(C P)^{2}=-1$, which show that the BdG Hamiltonian belongs to the $\mathbf{k}$-local symmetry class $\mathrm{CI}+\mathcal{I}$ [32]. In this class, 2D Dirac points or 3D nodal lines can be protected as in the case of the class $\mathrm{AI}+\mathcal{I}$. The only difference is that the $1 \mathrm{D}$ invariant is integer-valued in the class $\mathrm{CI}+\mathcal{I}$, but this is irrelevant in our analysis below because we are only interested in the parity of the 1D invariant that can be related to the eigenvalues of $P$.

Nodal structure of TSC and parity formula. According to Eq. (2), an odd-parity pairing function $\Delta(\mathbf{k})$ changes its sign on the Fermi surfaces surrounding a time-reversal-invariant momentum (TRIM) so that an even number of nodes should appear at the points where the sign of $\Delta(\mathbf{k})$ changes. The number of nodal points can be related with the inversion parities of occupied bands using the idea proposed in Refs. [6-8] as follows. In 2D, the parity of the number of Dirac node pairs related by inversion can be counted by the $Z_{2}$ invariant $v_{1} \equiv$ $\sum_{\mathbf{K} \in \text { TRIM }} n_{-}^{\text {o }}(\mathbf{K}) \bmod 2$ [34], where $n_{-}^{\mathrm{o}}(\mathbf{K})$ is the number of occupied states with negative parity at $\mathbf{K}$. Here, $v_{1}$ can be understood as the number of band inversions at TRIM that create pairs of Dirac points, starting from the trivial phase with only positive-parity occupied states.

One can define a similar parity index $v_{1}^{\mathrm{BdG}}$ for the BdG Hamiltonian as

$$
\begin{aligned}
v_{1}^{\mathrm{BdG}} & \equiv \sum_{\mathbf{K} \in \mathrm{TRIM}} n_{-}^{\mathrm{BdG} ; \mathrm{o}}(\mathbf{K}) \\
& =\sum_{\mathbf{K} \in \mathrm{TRIM}} n_{-}^{\mathrm{o}}(\mathbf{K})+n_{+}^{\mathrm{u}}(\mathbf{K}) \\
& =\sum_{\mathbf{K} \in \mathrm{TRIM}} n^{\mathrm{u}}(\mathbf{K}) \bmod 2,
\end{aligned}
$$

where $n_{ \pm}^{\mathrm{o}(\mathrm{u})}$ is the number of occupied (unoccupied) states with \pm parity in the normal state, $n^{\mathrm{u}}=n_{+}^{\mathrm{u}}+n_{-}^{\mathrm{u}}$, and $n_{ \pm}^{\mathrm{BdG} ; \mathrm{o}(\mathrm{u})}$ is defined similarly for the BdG Hamiltonian with an odd-parity pairing function. The second line in Eq. (3) results from the odd-parity pairing, and the third line follows from $n_{-}^{\text {o }}$ $(\mathbf{K})=n_{-}^{\mathrm{o}}(\mathbf{K})+n_{-}^{\mathrm{u}}(\mathbf{K})-n_{-}^{\mathrm{u}}(\mathbf{K})=n_{-}^{\mathrm{o}}(\mathbf{K})+n_{-}^{\mathrm{u}}(\mathbf{K})+n_{-}^{\mathrm{u}}(\mathbf{K})$ mod 2 together with $\sum_{\mathbf{K}} n_{-}(\mathbf{K})=0 \bmod 2$ following from that, when all the bands are occupied, and no band crossing exists at the Fermi level. Equation (3) shows that $v_{1}^{\mathrm{BdG}}=1$ mod 2 only when there exists an odd number of Fermi surfaces. This is consistent with the odd-parity condition of the pairing function $\Delta(\mathbf{k})$ on the Fermi surface in Eq. (2), which guarantees an odd number of Dirac node pairs in the superconducting state per each normal-state Fermi surface enclosing a TRIM.

Generalized parity formula for second-order TSC in 2D. To derive the condition for higher-order superconductivity of spin-polarized electrons, let us introduce generalized parity formulas. According to the Dirac Hamiltonian formalism for inversion-protected higher-order topological phases [9,35], we can obtain a higher-order TI by inverting $2^{n}$ bands at a TRIM starting from a topologically trivial phase. Here, $n$ denotes a non-negative integer. Therefore, counting the number of the simultaneous inversion of $2^{n}$ bands at TRIM 


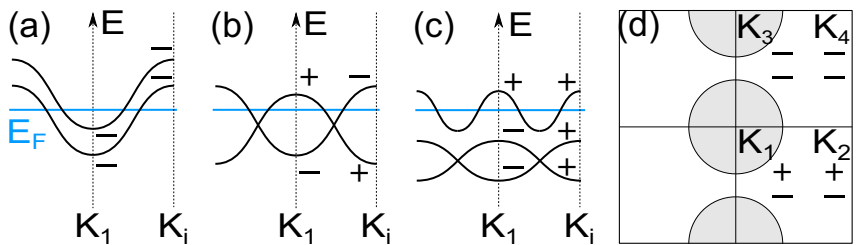

FIG. 1. Band structure and parity configuration of spin-polarized metals leading to 2D second-order TSCs in the weak-pairing limit. (a) Two electronlike (or holelike) Fermi surfaces surrounding the same TRIM. (b) Doped DSM with $v_{1}=1$. (c) Normal state whose whole bands, including both occupied and unoccupied bands, have the higher-order topology with $v_{2}=1$. The horizontal axes in (a)(c) schematically represent the $2 \mathrm{D}$ Brillouin zone: $\mathbf{K}_{1}=(0,0)$, and $\mathbf{K}_{i}$ indicates the other three TRIM with the same parity configuration. \pm represents the parity at TRIM. (d) The fourth way to obtain the higher-order TSCs. Here, the \pm sign on the top (bottom) row at each TRIM represents the parity of the higher-energy (lower-energy) states. One (no) band is occupied in the gray (white) regions, and the boundaries show the relevant Fermi surfaces.

leads to the following $Z_{2}$ index,

$$
v_{2^{n}} \equiv \sum_{\mathbf{K} \in \text { TRIM }}\left[\frac{n_{-}^{o}(\mathbf{K})}{2^{n}}\right]_{\text {floor }} \bmod 2,
$$

where $[m+a]_{\text {floor }}=m$ for an integer $m$ and $0 \leqslant a<1$. We can also introduce similar indices $\nu_{2^{n}}^{\mathrm{BdG}}$ for the BdG Hamiltonian by replacing $n_{-}^{\mathrm{o}}(\mathbf{K})$ by $n_{-}^{\mathrm{BdG} ; \mathrm{o}}(\mathbf{K})$. These indices characterize higher-order TSCs.

Let us first discuss the physical meaning of $v_{2}^{\mathrm{BdG}}$ in 2D. Recently, it was shown that $v_{2}=1$ indicates the second-order topology of a $P T$-symmetric topological insulator with chiral symmetry, characterized by fractional corner charges on the boundary [35-37]. A straightforward extension of this idea shows that $v_{2}^{\text {BdG }}=1$ characterizes a second-order TSC with Majorana corner modes. Explicitly, $v_{2}^{\mathrm{BdG}}$ can be decomposed as

$$
\begin{aligned}
v_{2}^{\mathrm{BdG}}= & \sum_{\mathbf{K} \in \mathrm{TRIM}}\left[\frac{n^{\mathrm{u}}(\mathbf{K})}{2}\right]_{\text {floor }}+\sum_{\mathbf{K} \in \mathrm{TRIM}} n_{-}^{\mathrm{o}}(\mathbf{K}) \\
& +\sum_{\mathbf{K} \in \mathrm{TRIM}}\left[\frac{n_{-}(\mathbf{K})}{2}\right]_{\text {floor }}+\sum_{\mathbf{K} \in \mathrm{TRIM}} \delta_{2}(\mathbf{K}) \bmod 2,
\end{aligned}
$$

where $\delta_{2}(\mathbf{K})=\left[n^{\mathrm{u}}(\mathbf{K})+1\right] n_{-}(\mathbf{K}) \bmod 2$. The detailed derivation is in the Supplemental Material [33]. In Eq. (5), the first term counts the parity of the number of "double Fermi surfaces," that is, two electronlike (or holelike) Fermi surfaces enclosing the same TRIM, in the normal state. The second term is $v_{1}$ for the occupied bands in the normal state and the third term is $v_{2}$ when all bands are occupied in the normal state. Finally, the last term counts the number of TRIM with an even number of unoccupied states and an odd number of negative-parity eigenstates. Figures 1(a)-1(d) show four different normal-state band structures leading to $v_{2}^{\mathrm{BdG}}=1$ in the weak-pairing limit, which arise from the nontrivial value of the first, second, third, and fourth terms in Eq. (5), respectively.

The analysis of Eq. (5) becomes much simpler in systems with an inversion-symmetric unit cell, where all atoms in a unit cell can be adiabatically shifted to its center without breaking inversion symmetry. In this case, the third term in Eq. (5) vanishes because an inversion-symmetric unit cell gives a topologically trivial state with $v_{2}=1$ when all bands are occupied. Similarly, the zero Berry phase of the whole bands makes the fourth term vanish (see Supplemental Material [33]).

Then, there remain two different channels leading to $v_{2}^{\mathrm{BdG}}=1$ : One is odd-parity pairing in a metal with double Fermi surfaces, and the other is odd-parity pairing in a doped DSM, whose nontrivial band topology arises from the first and second terms in Eq. (5), respectively. In general, the former induces nodal superconductivity rather than a fully gapped TSC. This is because each of the two Fermi surfaces encloses a TRIM so that an odd-parity pairing function accompanies the sign reversal at two points on the Fermi surface, generating Dirac nodes. A strong pairing is required to get a fully gapped superconducting state via pair annihilations of Dirac nodes, unless the system is fine tuned so that the two Fermi surfaces are very close to each other. On the other hand, even weak pairing generates a fully gapped superconducting state in doped DSMs because two disconnected Fermi surfaces, each centered at a generic momentum, are paired in this case.

Higher-order TSCs in 3D and further generalization. In 3D, $v_{1}=1$ indicates an odd number of nodal lines [34], and $v_{2}=$ 1 indicates an odd number of pairs of monopole nodal lines in the Brillouin zone [31,38]. Similarly, $v_{1}^{\mathrm{BdG}}=1\left(v_{2}^{\mathrm{BdG}}=1\right)$ indicates a superconductor with an odd number of nodal lines (monopole nodal line pairs). In particular, the superconductor with a monopole nodal line pair exhibits the second-order topological property and carries anomalous hinge Majorana states, as in the case of chiral-symmetric monopole NLSMs [36]. Similar to 2D cases, the most promising way to get $v_{2}^{\mathrm{BdG}}=1$ is the process with a nontrivial second term in Eq. (5), which corresponds to doping spin-polarized NLSMs. The third term in Eq. (5) always vanishes when the whole bands are fully considered. Also the fourth term vanishes if we take an inversion-symmetric unit cell as in $2 \mathrm{D}$. In the case of the first term, it may be relevant in a strong-pairing limit. A double Fermi surface normally generates a superconducting state with nodal lines carrying trivial monopole charges from each Fermi surface. When the pairing amplitude is sufficiently strong, however, the two trivial nodal lines may recombine and turn into two monopole nodal lines. We note that the same mechanism corresponding to the second term in Eq. (5) was also proposed in Ref. [32] for systems with SU(2) spin rotation symmetry.

The above formulation can be generalized further to $v_{2^{n}}^{\mathrm{BdG}}$ with an arbitrary $n$,

$$
\begin{aligned}
v_{2^{n}}^{\mathrm{BdG}}= & \sum_{\mathbf{K} \in \mathrm{TRIM}}\left[\frac{n^{\mathrm{u}}(\mathbf{K})}{2^{n}}\right]_{\text {floor }}+\sum_{\mathbf{K} \in \mathrm{TRIM}}\left[\frac{n_{-}^{\mathrm{o}}(\mathbf{K})}{2^{n-1}}\right]_{\text {floor }} \\
& +\sum_{\mathbf{K} \in \mathrm{TRIM}}\left[\frac{n_{-}(\mathbf{K})}{2^{n}}\right]_{\text {floor }}+\sum_{\mathbf{K} \in \mathrm{TRIM}} \delta_{2^{n}}(\mathbf{K}) \bmod 2,
\end{aligned}
$$

where the definition of $\delta_{2^{n}}(\mathbf{K})$ is given in the Supplemental Material [33]. In particular, $v_{4}^{\mathrm{BdG}}=1$ characterizes the third-order TSC in 3D [35]. By the same reason discussed above, one can show that the best way to get a fully gapped 
superconductivity with $v_{4}^{\mathrm{BdG}}=1$ is to use the process related with the second term in Eq. (6), which can be achieved by doping a monopole NLSM (see the Supplemental Material for details [33]). To sum up, in ferromagnetic systems with an inversion-symmetric unit cell, doped nodal semimetals are the best normal state to get a higher-order TSC in the weakpairing limit.

Lattice model. We demonstrate our theory by using simple tight-binding models defined on rectangular or orthorhombic lattices. We construct three models in which the spinpolarized normal states are a 2D DSM, a 3D NLSM, and a 3D monopole NLSM, respectively. When an odd-parity superconducting pairing is introduced, we show that the three nodal semimetals turn into a 2D second-order TSC, a 3D monopole nodal-line superconductor, and a 3D third-order TSC, respectively.

First, a 2D DSM can be described by the nearest-neighbor tight-binding Hamiltonian for $s$ and $p_{x}$ orbitals as

$$
h=-\mu+2 t \sin k_{x} \sigma_{y}+\left(M-2 t \cos k_{x}-2 t \cos k_{y}\right) \sigma_{z},
$$

where the Pauli matrices $\sigma_{y, z}$ describe the orbital degrees of freedom with $\uparrow(\downarrow)$ indicating an $s\left(p_{x}\right)$ orbital. The corresponding band structure exhibits two Dirac points on the $k_{x}=0$ line when $0<M / t<4$ at the energy $E=-\mu$. To induce superconductivity, we consider the following interaction term, $H_{\text {int }}=-U \sum_{i, \sigma \neq \sigma^{\prime}} n_{i, \sigma} n_{i, \sigma^{\prime}}-V \sum_{\langle i, j\rangle, \sigma} n_{i, \sigma} n_{j, \sigma}$, where $U(V)$ indicates the on-site interorbital (nearest-neighbor intraorbital) interaction, which is to be treated by a meanfield approximation. The resulting odd-parity pairing leads to a fully gapped TSC whose second-order band topology is clearly demonstrated in Figs. 2(a) and 2(b). Vertically stacking the 2D DSM and introducing interlayer hopping, described by $-2 t \cos k_{z} \sigma_{z}$, we obtain the Hamiltonian for a 3D NLSM. Also, by further adding $p_{y}$ and $d_{x y}$ orbitals at each lattice site and introducing nearest-neighbor hopping, we obtain a 3D monopole NLSM. Adding an odd-parity pairing function in these NLSMs leads to a 3D monopole nodal-line superconductor and a 3D third-order TSC whose topological properties are demonstrated in Figs. 2(c)-2(f). Detailed information about the tight-binding models is given in the Supplemental Material [33].

Discussions. We first discuss the effect of the inversion asymmetry of the unit cell. For instance, in the kagome lattice, the unit cell always breaks inversion symmetry if all atoms are required to be strictly within the unit cell. One may choose a unit cell, invariant under inversion up to lattice translations, only when the atoms in a unit cell are located on its boundary. In this case, $v_{2}=1$ when each atom is occupied by one electron, so the third term in Eq. (5) is nontrivial [33] for a threeband tight-binding model. Then, we have $\nu_{2}^{\mathrm{BdG}}=0$ even when the normal state is a doped DSM. However, this does not mean that MZM is absent on the boundary. In fact, one can show that MZMs exist (do not exist) when $v_{2}^{\text {BdG }}=0\left(v_{2}^{\text {BdG }}=1\right)$, in contrast to systems having an inversion-symmetric unit cell. To obtain more conventional bulk-boundary correspondence where $v_{2}^{\mathrm{BdG}}=1$ always indicates the existence of MZMs independent of symmetry of the unit cell, one may define a reference trivial phase of the TSC as the limit $\mu \rightarrow-\infty$ where all electrons are unoccupied in the normal state as proposed in Ref. [39]. This gives a well-defined trivial phase

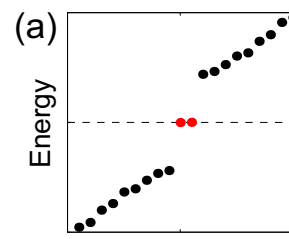

States

(b)
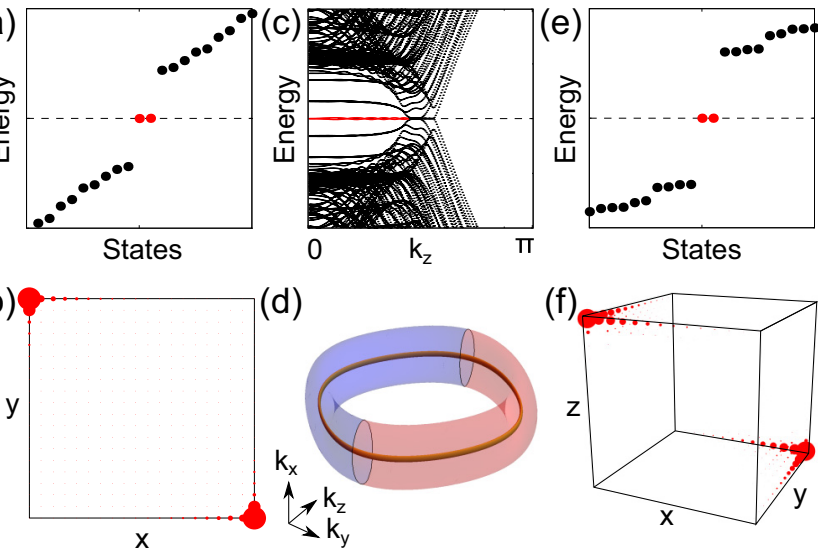

States

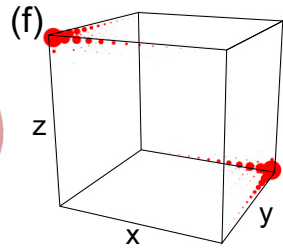

FIG. 2. Higher-order topological superconductivity from lattice models. (a), (b) 2D second-order TSC obtained by adding an oddparity pairing function to the doped 2D DSM described in Eq. (7). (a) Energy spectrum on a finite-size lattice. (b) Probability density of a Majorana zero mode. (c), (d) Monopole nodal-line superconductor derived from a doped 3D NLSM. (c) Energy spectrum of the system, finite-sized along the $x$ and $y$ directions. (d) Nodal structure in the Brillouin zone. The torus indicates the Fermi surface enclosing a nodal line (thick gold line) in the normal state. The blue (red) color indicates the region where the pairing function has positive (negative) sign. Two monopole nodal loops appear at the intersection, where the pairing function changes its sign. (e), (f) 3D third-order TSC derived from a doped 3D monopole NLSM. (e) Energy spectrum on a finite-size lattice. (f) Probability density of a Majorana zero mode.

for TSCs because Majorana fermions are confined to form electrons in such a limit: $|\mu|$ serves as the binding energy for Majorana fermions because $\mu c_{\mathbf{x}}^{\dagger} c_{\mathbf{x}}=2 i \mu \gamma_{1 \mathbf{x}} \gamma_{2 \mathbf{x}}$ at each site $\mathbf{x}$ [40], where Majorana operators $\gamma_{1,2}$ are defined from the electron annihilation operator $c_{\mathbf{x}}=\gamma_{1 \mathbf{x}}+i \gamma_{2 \mathbf{x}}$.

Next, let us discuss the effect of spin-orbit coupling. When spin-orbit coupling is included, $T$ symmetry is broken because the electron's spin cannot rotate freely independent of the orbital degrees of freedom. Since the protection of the nodal structures in both normal and superconducting states requires the combination of time-reversal and inversion symmetries, the nodal structures become unstable when spin-orbit coupling exists. However, our formula in Eq. (6) is still applicable as long as inversion symmetry is preserved. Accordingly, a gapped higher-order TSC can still survive if the parity configuration does not change due to spin-orbit coupling, since their topology can be protected by inversion symmetry only. In the case of the monopole nodal-line superconductor, the nodes are fully gapped when $T$ symmetry is broken due to spin-orbit coupling. The resulting gapped superconductor is a secondorder TSC hosting chiral hinge states $[9,31,41]$. In fact, in the normal state, NLSM transforms to a Weyl semimetal by spin-orbit coupling as long as the parity configuration does not change. This means that, when spin-orbit coupling exists, what we observe is the transition from a Weyl semimetal to a fully gapped second-order TSC.

There exist many candidate materials for ferromagnetic spin-polarized 2D Dirac semimetals and some 3D nodalline semimetals [42-47]. One way to realize spin-triplet pairing in $2 \mathrm{D}$ ferromagnetic nodal semimetals is to use a 
superconductor-ferromagnet-superconductor heterostructure with inversion symmetry. Here, we can use conventional spinsinglet $s$-wave superconductors and a ferromagnet with inplane magnetization. After spin-singlet Cooper pairs penetrate into the ferromagnet, they can turn into spin-triplet Cooper pairs because of the spin polarization in the ferromagnet [48]. In $3 \mathrm{D}$, on the other hand, an intrinsic superconducting pairing is required because the proximity effect is not effective. In fact, there are several materials where the coexistence of ferromagnetism and superconductivity is reported, including uranium-based materials $\mathrm{UGe}_{2}, \mathrm{URhGe}, \mathrm{UCoGe}, \mathrm{UTe}_{2}$ [49-55], and the more recently proposed twisted doublebilayer graphene [56-58]. We hope that our work stimulates the research on higher-order TSC in ferromagnets. This will open another route to Majorana quantum computations, where ferromagnetic nodal semimetals with a spin-polarized band crossing serve as platforms for higher-order TSCs.

Finally, let us briefly comment on the extension of our result to other symmetry classes. We note that our parity formula Eq. (4) is generally applicable to any odd-parity superconductors, while we focus on ferromagnetic systems with effective time-reversal symmetry since odd-parity pairing is natural in these systems. Furthermore, we expect that nodal semimetals required by eigenvalues of symmetry operator $G$ can lead to a $G$-protected $d$ th-order TSCs, which can be shown by extending Eq. (4) to eigenvalues of $G$, as is outlined in Ref. [29] for $k$ th-order TSCs with $k<d$. We leave a more detailed theoretical analysis for future work.

Note added. Recently, we became aware of two related articles [39,59]. Reference [59] also suggests that a third-order topological superconductivity can be obtained by odd-parity pairing in a doped nodal-line semimetal. In Ref. [39], the authors also have related the higher-order topology of inversionsymmetric superconductors to the parity of the normal state.

Acknowledgments. We are grateful to A. Skurativska, T. Neupert, and M. H. Fischer for sharing a related manuscript [39]. We thank Seunghun Lee, Yoonseok Hwang, Taekoo Oh, and Se Young Park for helpful discussions. J.A. was supported by IBS-R009-D1. B.-J.Y. was supported by the Institute for Basic Science in Korea (Grant No. IBS-R009-D1) and Basic Science Research Program through the National Research Foundation of Korea (NRF) (Grant No. 0426-20190008), and the POSCO Science Fellowship of POSCO TJ Park Foundation (No. 0426-20180002). This work was supported in part by the U.S. Army Research Office under Grant No. W911NF18-1-0137.
[1] M. Sato and Y. Ando, Topological superconductors: A review, Rep. Prog. Phys. 80, 076501 (2017).

[2] J. Alicea, New directions in the pursuit of Majorana fermions in solid state systems, Rep. Prog. Phys. 75, 076501 (2012).

[3] S. Das Sarma, M. Freedman, and C. Nayak, Majorana zero modes and topological quantum computation, npj Quantum Inf. 1, 15001 (2015).

[4] A. Kitaev, Fault-tolerant quantum computation by anyons, Ann. Phys. 303, 2 (2003)

[5] C. Nayak, S. H. Simon, A. Stern, M. Freedman, and S. Das Sarma, Non-Abelian anyons and topological quantum computation, Rev. Mod. Phys. 80, 1083 (2008).

[6] L. Fu and E. Berg, Odd-Parity Topological Superconductors: Theory and Application to $\mathrm{Cu}_{x} \mathrm{Bi}_{2} \mathrm{Se}_{3}$, Phys. Rev. Lett. 105, 097001 (2010).

[7] M. Sato, Topological properties of spin-triplet superconductors and Fermi surface topology in the normal state, Phys. Rev. B 79, 214526 (2009).

[8] M. Sato, Topological odd-parity superconductors, Phys. Rev. B 81, 220504(R) (2010).

[9] E. Khalaf, Higher-order topological insulators and superconductors protected by inversion symmetry, Phys. Rev. B 97, 205136 (2018).

[10] Y. You, D. Litinski, and F. von Oppen, Higher order topological superconductors as generators of quantum codes, Phys. Rev. B 100, 054513 (2019).

[11] M. Geier, L. Trifunovic, M. Hoskam, and P. W. Brouwer, Second-order topological insulators and superconductors with an order-two crystalline symmetry, Phys. Rev. B 97, 205135 (2018).

[12] L. Trifunovic and P. W. Brouwer, Higher-Order Bulk-Boundary Correspondence for Topological Crystalline Phases, Phys. Rev. X 9, 011012 (2019).

[13] Y. Wang, M. Lin, and T. L. Hughes, Weak-pairing higher order topological superconductors, Phys. Rev. B 98, 165144 (2018).
[14] T. E. Pahomi, M. Sigrist, and A. A. Soluyanov, Braiding Majorana corner modes in a two-layer second-order topological insulator, arXiv:1904.07822.

[15] T. Liu, J. J. He, F. Nori et al., Majorana corner states in a two-dimensional magnetic topological insulator on a hightemperature superconductor, Phys. Rev. B 98, 245413 (2018).

[16] Q. Wang, C.-C. Liu, Y.-M. Lu, and F. Zhang, High-Temperature Majorana Corner States, Phys. Rev. Lett. 121, 186801 (2018).

[17] Z. Yan, F. Song, and Z. Wang, Majorana Corner Modes in a High-Temperature Platform, Phys. Rev. Lett. 121, 096803 (2018).

[18] C.-H. Hsu, P. Stano, J. Klinovaja, and D. Loss, Majorana Kramers Pairs in Higher-Order Topological Insulators, Phys. Rev. Lett. 121, 196801 (2018).

[19] X. Zhu, Second-Order Topological Superconductors with Mixed Pairing, Phys. Rev. Lett. 122, 236401 (2019).

[20] K. Laubscher, D. Loss, and J. Klinovaja, Fractional topological superconductivity and parafermion corner states, Phys. Rev. Research 1, 032017(R) (2019).

[21] X. Zhu, Tunable Majorana corner states in a two-dimensional second-order topological superconductor induced by magnetic fields, Phys. Rev. B 97, 205134 (2018).

[22] Y. Volpez, D. Loss, and J. Klinovaja, Second-Order Topological Superconductivity in $\pi$-Junction Rashba Layers, Phys. Rev. Lett. 122, 126402 (2019).

[23] Y.-T. Hsu, W. S. Cole, R.-X. Zhang, and J. D. Sau, Inversionprotected topological crystalline superconductivity in monolayer $\mathrm{WTe}_{2}$, arXiv:1904.06361.

[24] Y.-J. Wu, J. Hou, Y.-M. Li, X.-W. Luo, and C. Zhang, In-plane Zeeman field induced Majorana corner and hinge modes in an $s$-wave superconductor heterostructure, arXiv:1905.08896.

[25] R.-X. Zhang, W. S. Cole, X. Wu, and S. Das Sarma, Higher Order Topology and Nodal Topological Superconductivity in $\mathrm{Fe}(\mathrm{Se}, \mathrm{Te})$ Heterostructures, Phys. Rev. Lett. 123, 167001 (2019). 
[26] Z. Wu, Z. Yan, and W. Huang, Higher-order topological superconductivity: Possible realization in Fermi gases and $\mathrm{Sr}_{2} \mathrm{RuO}_{4}$, Phys. Rev. B 99, 020508(R) (2019).

[27] S. Franca, D. V. Efremov, and I. C. Fulga, Phase tunable secondorder topological superconductor, Phys. Rev. B 100, 075415 (2019).

[28] M. Kheirkhah, Y. Nagai, C. Chen, and F. Marsiglio, Majorana corner flat bands in two-dimensional second-order topological superconductors, Phys. Rev. B 101, 104502 (2020).

[29] S. Ono, Y. Yanase, and H. Watanabe, Symmetry indicators for topological superconductors, Phys. Rev. Research 1, 013012 (2019).

[30] C. Fang and L. Fu, New classes of three-dimensional topological crystalline insulators: Nonsymmorphic and magnetic, Phys. Rev. B 91, 161105(R) (2015).

[31] J. Ahn, D. Kim, Y. Kim, and B.-J. Yang, Band Topology and Linking Structure of Nodal Line Semimetals with $Z_{2}$ Monopole Charges, Phys. Rev. Lett. 121, 106403 (2018).

[32] T. Bzdušek and M. Sigrist, Robust doubly charged nodal lines and nodal surfaces in centrosymmetric systems, Phys. Rev. B 96, 155105 (2017).

[33] See Supplemental Material at http://link.aps.org/supplemental/ 10.1103/PhysRevResearch.2.012060 for the derivation of the generalized parity formula and tight-binding models of spinpolarized higher-order superconductors.

[34] Y. Kim, B. J. Wieder, C. L. Kane, and A. M. Rappe, Dirac Line Nodes in Inversion-Symmetric Crystals, Phys. Rev. Lett. 115, 036806 (2015).

[35] Y. Hwang, J. Ahn, and B.-J. Yang, Fragile topology protected by inversion symmetry: Diagnosis, bulk-boundary correspondence, and Wilson loop, Phys. Rev. B 100, 205126 (2019).

[36] Z. Wang, B. J. Wieder, J. Li, B. Yan, and B. A. Bernevig, Higher-Order Topology, Monopole Nodal Lines, and the Origin of Large Fermi Arcs in Transition Metal Dichalcogenides $X \mathrm{Te}_{2}$ ( $X=$ Mo, W), Phys. Rev. Lett. 123, 186401 (2019).

[37] J. Ahn, S. Park, and B.-J. Yang, Failure of Nielsen-Ninomiya theorem and Fragile Topology in Two-Dimensional Systems with Space-Time Inversion Symmetry: Application to Twisted Bilayer Graphene at Magic Angle, Phys. Rev. X 9, 021013 (2019).

[38] Z. Song, T. Zhang, and C. Fang, Diagnosis for Nonmagnetic Topological Semimetals in the Absence of Spin-Orbital Coupling, Phys. Rev. X 8, 031069 (2018).

[39] A. Skurativska, T. Neupert, and M. H. Fischer, Atomic limit and inversion-symmetry indicators for topological superconductors, Phys. Rev. Research 2, 013064 (2020).

[40] A. Y. Kitaev, Unpaired Majorana fermions in quantum wires, Phys. Usp. 44, 131 (2001).

[41] E. Khalaf, H. C. Po, A. Vishwanath, and H. Watanabe, Symmetry Indicators and Anomalous Surface States of Topological Crystalline Insulators, Phys. Rev. X 8, 031070 (2018).

[42] X. Wang, T. Li, Z. Cheng, X.-L. Wang, and H. Chen, Recent advances in Dirac spin-gapless semiconductors, Appl. Phys. Rev. 5, 041103 (2018).

[43] J. Zou, Z. He, and G. Xu, The study of magnetic topological semimetals by first principles calculations, npj Comput. Mater. 5, 1 (2019).
[44] R. Wang, J. Z. Zhao, Y. J. Jin, Y. P. Du, Y. X. Zhao, H. Xu, and S. Y. Tong, Nodal line fermions in magnetic oxides, Phys. Rev. B 97, 241111(R) (2018)

[45] K. Kim, J. Seo, E. Lee, K.-T. Ko, B. Kim, B. G. Jang, J. M. Ok, J. Lee, Y. J. Jo, W. Kang et al., Large anomalous Hall current induced by topological nodal lines in a ferromagnetic van der Waals semimetal, Nat. Mater. 17, 794 (2018).

[46] C. Chen, Z.-M. Yu, S. Li, Z. Chen, X.-L. Sheng, and S. A. Yang, Weyl-loop half-metal in $\mathrm{Li}_{3}\left(\mathrm{FeO}_{3}\right)_{2}$, Phys. Rev. B 99, 075131 (2019).

[47] X. Zuo, A. C. Dias, F. Liu, L. Han, H. Li, Q. Gao, X. Jiang, D. Li, B. Cui, D. Liu, F. Qu, Fully spin-polarized open and closed nodal lines in $\beta$-borophene by magnetic proximity effect, Phys. Rev. B 100, 115423 (2019).

[48] M. Eschrig, J. Kopu, J. C. Cuevas, and G. Schön, Theory of Half-Metal/Superconductor Heterostructures, Phys. Rev. Lett. 90, 137003 (2003).

[49] D. Aoki, K. Ishida, and J. Flouquet, Review of U-based ferromagnetic superconductors: Comparison between $\mathrm{UGe}_{2}$, URhGe, and UCoGe, J. Phys. Soc. Jpn. 88, 022001 (2019).

[50] S. S. Saxena, P. Agarwal, K. Ahilan, F. M. Grosche, R. K. W. Haselwimmer, M. J. Steiner, E. Pugh, I. R. Walker, S. R. Julian, P. Monthoux et al., Superconductivity on the border of itinerantelectron ferromagnetism in $\mathrm{UGe}_{2}$, Nature (London) 406, 587 (2000)

[51] A. Huxley, I. Sheikin, E. Ressouche, N. Kernavanois, D. Braithwaite, R. Calemczuk, and J. Flouquet, $\mathrm{UGe}_{2}$ : A ferromagnetic spin-triplet superconductor, Phys. Rev. B 63, 144519 (2001).

[52] D. Aoki, A. Huxley, E. Ressouche, D. Braithwaite, J. Flouquet, J.-P. Brison, E. Lhotel, and C. Paulsen, Coexistence of superconductivity and ferromagnetism in URhGe, Nature (London) 413, 613 (2001).

[53] N. T. Huy, A. Gasparini, D. E. De Nijs, Y. Huang, J. C. P. Klaasse, T. Gortenmulder, A. de Visser, A. Hamann, T. Görlach, and H. v. Löhneysen, Superconductivity on the Border of Weak Itinerant Ferromagnetism in UCoGe, Phys. Rev. Lett. 99, 067006 (2007).

[54] A. D. Huxley, Ferromagnetic superconductors, Physica C 514, 368 (2015).

[55] S. Ran, C. Eckberg, Q.-P. Ding, Y. Furukawa, T. Metz, S. R Saha, I.-L. Liu, M. Zic, H. Kim, J. Paglione et al., Nearly ferromagnetic spin-triplet superconductivity, Science 365, 684 (2019).

[56] X. Liu, Z. Hao, E. Khalaf, J. Y. Lee, K. Watanabe, T. Taniguchi, A. Vishwanath, and P. Kim, Spin-polarized correlated insulator and superconductor in twisted double bilayer graphene, arXiv: 1903.08130

[57] J. Y. Lee, E. Khalaf, S. Liu, X. Liu, Z. Hao, P. Kim, and A. Vishwanath, Theory of correlated insulating behaviour and spin-triplet superconductivity in twisted double bilayer graphene, Nat. Commun. 10, 5333 (2019).

[58] C. Shen, N. Li, S. Wang, Y. Zhao, J. Tang, J. Liu, J. Tian, Y. Chu, K. Watanabe, T. Taniguchi et al., Observation of superconductivity with $T_{c}$ onset at $12 \mathrm{~K}$ in electrically tunable twisted double bilayer graphene, arXiv:1903.06952.

[59] Z. Yan, Higher-Order Topological Odd-Parity Superconductors, Phys. Rev. Lett. 123, 177001 (2019). 